\title{
Adaptive Array Processing based Wireless Energy Transmission for IoT Applications
}

\author{
Dr. Joy Chen, Lu-Tsou Yeh, \\ Department of Electrical Engineering, \\ Da-Yeh University, \\ Dacun, Changhua, \\ Taiwan.
}

\begin{abstract}
Rechargeable energy sources are essential for the extreme deployment of Internet-of-Things (IoT) sensors with the massive growth in smart systems. In order to meet these requirements, wireless energy transmission (WET) provides demand based power to the sensors. Temporary energy storage is done using supercapacitors. This overcomes the drawback of release of hazardous wastes released by IoT connected disposables after their working life. WET is made possible through adaptive array processing. The system consists of a transmitting side with multiple antennas and a receiving side with a programmable energy harvester. Several far-field adaptive processing schemes such as conventional beamformers, multiple sidelobe canceller (MSLC), multiple beam antenna system, regenerative hybrid array, digital beamformer, and generalized sidelobe canceller are tested and compared with the proposed modified beamforming model for superior performance. As the number of antennas increases, the gain increases. Gain and cumulative distribution function are analyzed over multiple distances for multiple iterations. The received signal strength indicator (RSSI) is also estimated to validate the performance of the proposed model.
\end{abstract}

Keywords: Internet of Things, wireless energy transmission, beamforming, adaptive array processing, antennas;

\section{Introduction}

Nikole Tesla introduced the concept of wireless energy transfer in the year 1899. The preliminary wireless energy transmission experiment was unsafe and insufficient for powering real time devices [1]. However, with the advancements in technology, the efficiency of wireless energy transmission is improved to a usable level. Far-field radio-frequency radiation and non-radiative near-field coupling are two significant approaches of wireless energy transfer. Magnetic resonance (MR) coupling and inductive coupling techniques are used in nearfield non-radiative coupling [2]. Millions of sensors are expected to be interconnected with the advancement of the fifth generation $(5 \mathrm{G})$ mobile communication. Restock-able energy sources are essential for these sensors in large scale deployment for Internet-of-Things (IoT) applications [3].

The batteries and other components with toxic elements must be recycled or disposed appropriately, the failure of which, may lead to environmental hazards that lead to bigger concerns. These challenges are overcome by wireless power transfer (WPT) and far-field radio frequency (RF) transmission [4]. The release of hazardous waste from sensors after their expiry can be avoided by performing temporary energy storage using supercapacitors and on demand sensor powering models. No significant research is found on digital beamforming and practical implementation of prototypes or theoretical framework for wireless power transfer [5].

WPT principle is commonly used in RFID where the reader transmits the radio signal which undergoes remodulation and backscattering by a passive tag. Product related data, serial number, unique identifiers and such static data is transmitted by the RFID tags for non-volatile storage. For the system to function, the tags must be close to the transmitter or reader [6]. A power amplifier or energy buffer can be used for extending the communication range and signal strength. Along with this, optimization of cost, performance and structure are essential. An electromagnetic signal traveling through a radio channel tends to lose a significant percentage of its energy. Operation within the line-of-sight helps mitigating these losses to a certain extent by directing the transfer of power towards the receiver.

\section{Literature Review}

Simultaneous wireless information and power transfer (SWIPT), WPT, and RF energy harvesting has attracted the interest of academicians and researchers on a large scale over the recent past [7]. Optimization, linklevel analysis, beamforming algorithms, waveform design and rectifier design models are developed on concrete theoretical basis. Optimization of RF to DC is performed while comparing harmonic signals, chaotic signals, orthogonal frequency-division multiplexing (OFDM) signals and other such signals. The diode non-linearity can be characterized mathematically using data-based curve-fitting or Shockley diode equation for approximation and rectifier modeling. The design of WTP signal in terms of transmit diversity and fading impact are analyzed in the 
absence of the channel state information (CSI) [8]. Implementation of robust beamforming at the transmission module and providing RF power for multiple non-linear sensors that are rectifier-based is done simultaneously during the occurrence of imperfect CSI.

The range of inductive coupling using Qi protocol is around 40mm only. Several researchers are working towards improving this range using multiple devices [9]. The transfer efficiency is improved by tuning the resonant frequency using two coils in MR coupling. IoT architecture cannot accommodate near-field techniques efficiently due to scaling issues. It is essential to ensure reception of power across both indoor and outdoor environment efficiently [10]. Long distance WPT is performed on a large scale using radiative far field under directive beamforming and non-directive RF techniques. The ambient radio waves from WiFi or cellular systems are used for powering devices using non-directive RF. Directive beamforming can overcome the drawbacks of free-space path loss. Data communication of the future $5 \mathrm{G}$ technologies may greatly depend on beamforming techniques [11].

\section{Proposed Model}

Adaptive array processing algorithms are used for powering IoT devices wirelessly. Different ways of implementations of these algorithms are explored. Successful wireless transmission of electromagnetic energy from multiple antennas to the energy harvester requires an appropriate hardware configuration. RFID backscattering technique has been widely used by several researchers [12]. However, utilization of multiple antennas for testbed generation and WPT has not been performed significantly. The weights of beamforming must be sharpened using power metric as the transmitter feedback signal while implementing a energy harvester. Figure 1 shows the flow of transmission with three antennas with universal software radio peripheral (USRP). The received signal strength indicator (RSSI) at the energy harvesting module determines the index of the weight vector with the help of a computational unit. The weights of the proceeding iterations are sharpened using a feedback signal to the transmission module.

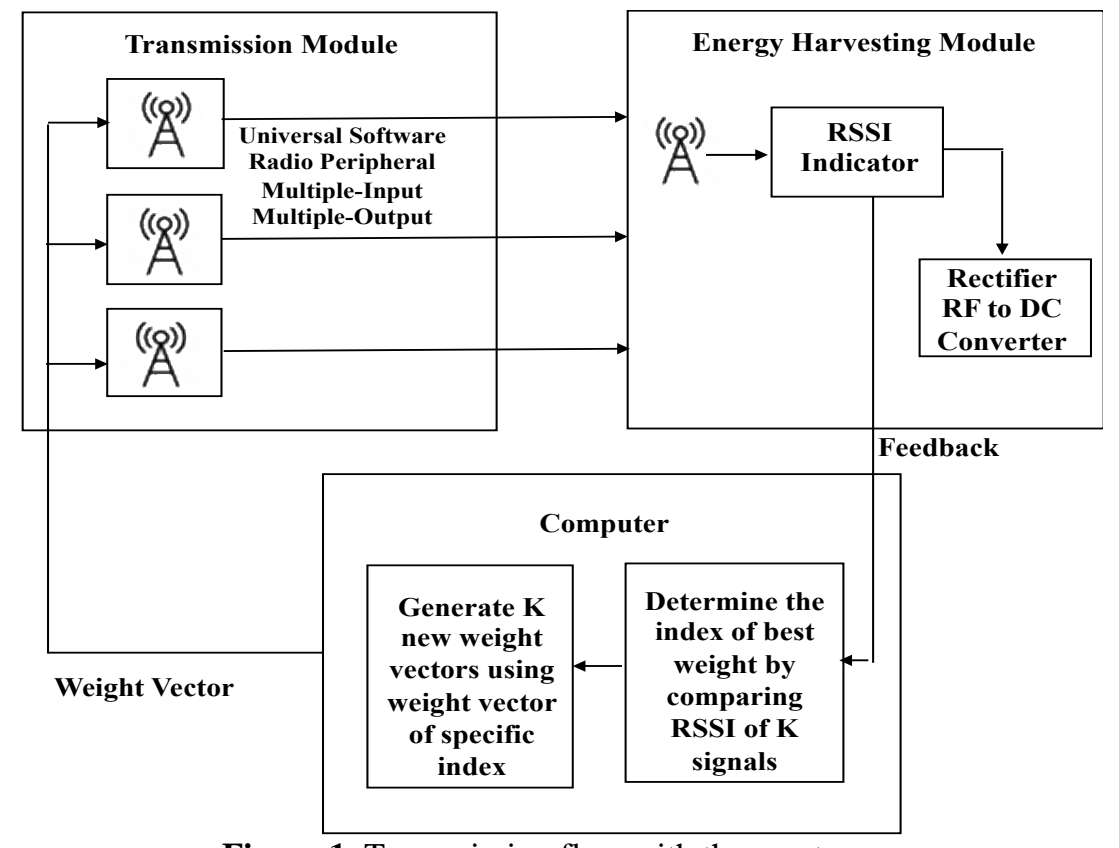

Figure 1. Transmission flow with three antennas

Multiple antennas of the range 2, 3, 4 and 6 are used for experimental purpose. An RF front end and FPGA is present in each antenna unit which acts as a software defined radio. A CPU is connected to the FPGA for signal processing. Python is used for programming the USRP units. A power-cast receiver (PR) receives the RSSI value transmitted using Zigbee protocol. A terminal emulation program, modem control and minicom are used for connecting a computer and PR for enabling feedback from the energy harvester. The weight vector index is analyzed in the computer using the RSSI data. The variations at the energy harvester of the RSSI circuit can be reduced considerably using a smoothing capacitor. In order to align the phase of each unit, synchronization of frequency and time are to be performed in beamforming systems with multiple antennas. 
The communication and power transmission can be turned off periodically to detect interference and prevent interference attacks. These attacks cause significant degradation in the charging performance. Dynamic frequency and power adjustment can protect the system from beamforming security attacks [13]. Other most common attacks include safety attacks, charging attacks like Free-rider, Greedy and Cheating energy receiver, interference attacks like beamforming and jamming attacks, spoofing attacks, application attacks and monitoring attacks [14]. Countermeasures like signature check, authentication, authorization, estimation of harvested energy, temperature estimation, RF exposure, periodic communication channel scan, interference alignment, scheduling dynamic frequency and power adjustment can help overcoming these security attacks [15].

\section{Simulation and Results}

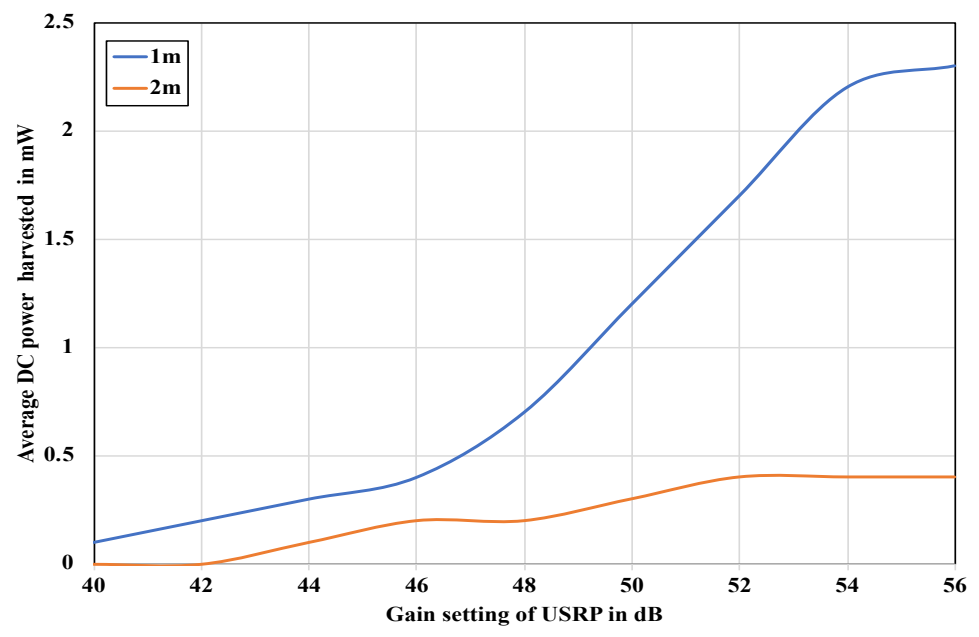

Figure 2. Average DC power harvested

The average harvested power, gain convergence and average RSSI values are analyzed for multiple antennas with multiple iteration and distance ranges. The strength of the power received improves substantially with the increase in number of iterations and number of antennas even over longer distances. The model is devised for receiving power signal at the energy harvester and measuring the received signal. Further, this signal is transmitted back. Several far-field adaptive processing schemes such as conventional beamformers, MSLC, multiple beam antenna system, regenerative hybrid array, digital beamformer, and generalized sidelobe canceller are tested and compared with the proposed modified beamforming model for superior performance. A testbed is setup to operate at $915 \mathrm{MHz}$ using the Universal Software Radio Peripheral (USRP). Figure 2 provides the average DC power harvested based on the average RF power that is transmitted. Higher the gain settings of USRP, greater the external power amplifier saturation. Energy is transmitted at a frequency of $915 \mathrm{MHz}$.

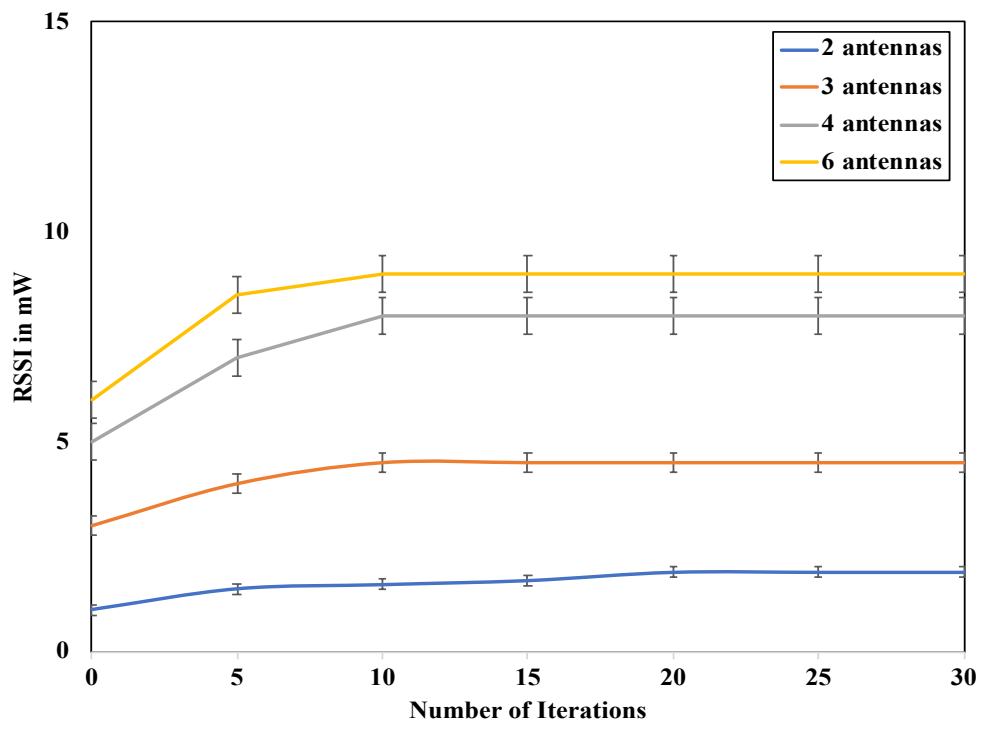

Figure 3. RSSI gain convergence 
Figure 3 represents the RSSI of gain convergence for multiple iterations. The four antenna configurations are tested with different weights. Same antenna configuration is run for all the far-field adaptive processing schemes such as conventional beamformers, MSLC, multiple beam antenna system, regenerative hybrid array, digital beamformer, and generalized sidelobe canceller. With the increase in the number of iterations, gain of the antenna increases. The convergence for each antenna setup is also higher. The signal consistency of the harvester also varies based on different algorithms used. Wireless power transfer networks are also prone to security attacks. It is essential to establish security goals and model to guarantee efficient and safe power transfer, availability, integrity, and to preserve sustainability. Further, random weights can be assigned to the signal for estimation of the RSSI distribution function.

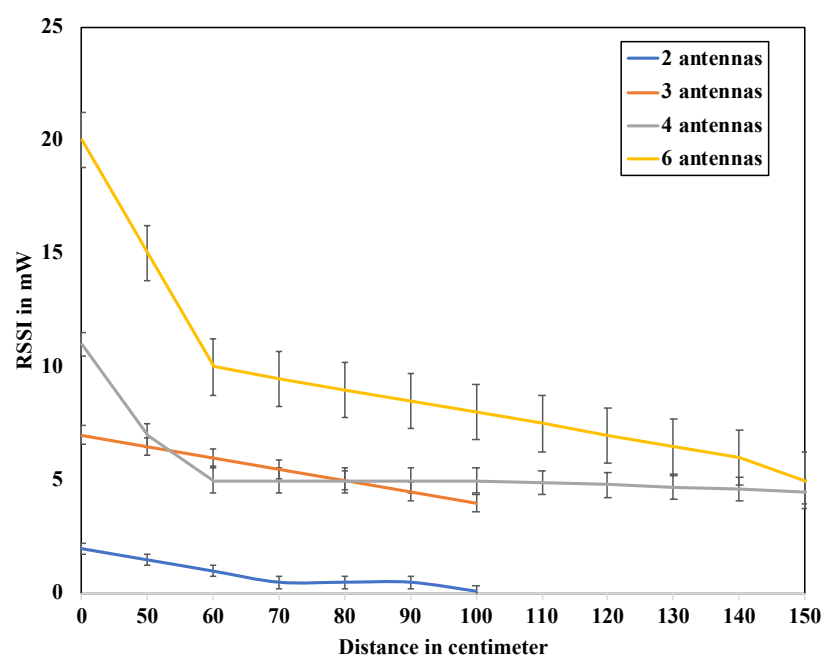

Figure 4. Average RSSI

The distance between the harvester and the transmitter is varied. The increase in number of iterations enable convergence to specific values and thereby increase the power received in contrast to the traditional techniques where the received power decreases with increase in distance. Figure 4 provides the average RSSI with increase in distance of the energy harvesting module. Constructive or destructive interference may occur when RF waves of same frequency band are transmitted by multiple energy transmitters at the same time. In certain cases, the energy harvesting operation can be intercepted to destroy the harvested energy leading to a complete system functionality collapse.

\section{Conclusion}

Beamforming is used for wireless energy transfer application on a practical as well as analytical level in this paper. Adaptive array processing schemes are discussed for efficient wireless energy transmission for IoT applications. Simple channel model and cost efficient estimation schemes are used for reducing computational cost. Continuous energy tones are transmitted simultaneously with common clock. This energy is received by the energy harvester for calculation of optimal RSSI signal. The average harvested power, gain convergence and average RSSI values are represented graphically for multiple antennas with multiple iteration and distance ranges. The strength of the power received improves substantially with the increase in number of iterations and number of antennas even over longer distances. The temperature sensors, motion sensors, wearable electronics, implantable sensors and other advanced sensors used in IoT devices that require smaller scale of power can be charged wirelessly using this technology. Future work is directed towards optimizing the model with lesser number of antennas and achieving enhanced power reception.

\section{References}

[1] Belo, D., Ribeiro, D. C., Pinho, P., \& Carvalho, N. B. (2019). A selective, tracking, and power adaptive far-field wireless power transfer system. IEEE Transactions on Microwave Theory and Techniques, 67(9), 3856-3866.

[2] Choi, K. W., Aziz, A. A., Setiawan, D., Tran, N. M., Ginting, L., \& Kim, D. I. (2018). Distributed wireless power transfer system for Internet of Things devices. IEEE Internet of Things Journal, 5(4), 2657-2671.

[3] Rana, M. M., Xiang, W., Wang, E., Li, X., \& Choi, B. J. (2018). Internet of Things infrastructure for wireless power transfer systems. IEEE Access, 6, 19295-19303. 
[4] Takacs, A., Okba, A., Aubert, H., Charlot, S., \& Calmon, P. F. (2017, May). Recent advances in electromagnetic energy harvesting and Wireless Power Transfer for IoT and SHM applications. In 2017 IEEE International Workshop of Electronics, Control, Measurement, Signals and their Application to Mechatronics (ECMSM) (pp. 1-4). IEEE.

[5] Fan, X., Ding, H., Li, S., Sanzari, M., Zhang, Y., Trappe, W., ... \& Howard, R. E. (2018). Energy-ball: Wireless power transfer for batteryless internet of things through distributed beamforming. Proceedings of the ACM on Interactive, Mobile, Wearable and Ubiquitous Technologies, 2(2), 1-22.

[6] Zhang, X., Zhang, X., \& Han, L. (2019). An energy efficient Internet of Things network using restart artificial bee colony and wireless power transfer. IEEE Access, 7, 12686-12695.

[7] Tran, H. V., \& Kaddoum, G. (2018). RF wireless power transfer: Regreening future networks. IEEE Potentials, 37(2), 35-41.

[8] Ejaz, W., Naeem, M., \& Zeadally, S. (2020). On-demand Sensing and Wireless Power Transfer for Selfsustainable Industrial IoT Networks. IEEE Transactions on Industrial Informatics.

[9] Helgesen, T., \& Haddara, M. (2018, November). Wireless power transfer solutions for 'things' in the internet of things. In Proceedings of the Future Technologies Conference (pp. 92-103). Springer, Cham.

[10] Pardue, C., Davis, A., Bellaredj, M., \& Swaminathan, M. (2017, November). Wireless power transfer integrated board for low power IoT applications. In 2017 IEEE Conference on Technologies for Sustainability (SusTech) (pp. 1-6). IEEE.

[11] Yanagawa, S., Shimizu, R., Hamada, M., Shimizu, T., \& Kuroda, T. (2017, November). Wireless power transfer to stacked modules for IoT sensor nodes. In 2017 International SoC Design Conference (ISOCC) (pp. 59-60). IEEE.

[12]Zhang, Q., Fang, W., Xiong, M., Liu, Q., Wu, J., \& Xia, P. (2018). Adaptive resonant beam charging for intelligent wireless power transfer. IEEE Internet of Things Journal, 6(1), 1160-1172.

[13] Smys, S., \& Wang, H. ENHANCED WIRELESS POWER TRANSFER SYSTEM FOR IMPLANTABLE MEDICAL DEVICES.

[14] Raj, J. S. (2019). QoS optimization of energy efficient routing in IoT wireless sensor networks. Journal of ISMAC, 1(01), 12-23.

[15] Sakya, S. (2020). Design of Hybrid Energy Management System for Wireless Sensor Networks in Remote Areas. Journal of Electrical Engineering and Automation (EEA), 2(01), 13-24. 